\title{
NONCONTINUITY OF TOPOLOGICAL ENTROPY OF MAPS OF THE CANTOR SET AND OF THE INTERVAL
}

\author{
LOUIS BLOCK
}

ABSTRACT. We show that topological entropy, as a map on the space of continuous functions of the Cantor set into itself, is not continuous anywhere. Furthermore, topological entropy, as a map on the space of continuous functions of the interval into itself, is not continuous at any map with finite entropy.

1. Introduction. For a compact topological space $S$, let $C^{0}(S, S)$ denote the space of continuous functions of $S$ into itself with the topology of uniform convergence. Let $I$ denote the unit interval $[0,1]$ and $C$ the Cantor set (the usual middle third Cantor set). For $f \in C^{0}(S, S)$, let ent $(f)$ denote the topological entropy of $f$ as defined in [1]. (We review the definition in $\S 2$.) ent $(f)$ is a nonnegative real number, or $\infty$, which describes (quantitatively) the action of $f$ considered as a discrete dynamical system.

Our main results are the following:

Theorem A. The function ent: $C^{0}(C, C) \rightarrow R \cup\{\infty\}$ is not continuous anywhere.

Theorem B. The function ent: $C^{0}(I, I) \rightarrow R \cup\{\infty\}$ is not continuous at any map $f$ with ent $(f)$ finite.

We note that Theorem $B$ is valid with $I$ replaced by the circle $S^{1}$. (See remarks at the end of $\$ 4$.)

Topological entropy has been studied in [4], [5] and [7] in connection with Smale's program [8] for studying the orbit structure of differentiable maps of manifolds. However the definition and basic properties rely only on continuity (see [1]). Thus it seems natural to determine what is true in the continuous case before proceeding to the differentiable case.

There are examples on higher dimensional manifolds (see [6]) to show that entropy is not continuous in the differentiable case. However, for the

Received by the editors April 5, 1974.

AMS (MOS) subject classifications (1970). Primary 54H20; Secondary 58F99.

Key words and phrases. Topological entropy, nonwandering set. 
circle or the interval the problem is open. In this connection we mention the following. Let $C^{1}(M, M)$ denote the space of continuously differentiable maps of a compact manifold $M$ into itself with the $C^{1}$ topology.

Theorem C. The function ent: $C^{1}(M, M) \rightarrow R$ is continuous at the identity map of $M$.

This follows from Proposition 12 of [5].

Finally we remark that positive results on continuity of entropy would have obvious consequences in the theory of bifurcations of differentiable maps. See [2] for some results in this direction.

2. Preliminary definitions and results. We begin by reviewing the definition of topological entropy as defined in [1]. Let $X$ be a compact topological space. For any two open covers $\mathbb{Q}$ and $\mathbb{B}$ of $X$, let $\mathbb{Q} \vee \mathfrak{B}$ denote $\{A \cap B \mid A \in \mathbb{P}$ and $B \in \mathfrak{B}\}$. Let $N(\mathbb{P})$ denote the number of sets in a subcover of $\oplus$ of minimum cardinality.

Let $f \in C^{0}(X, X)$. For each integer $n>0$ let

$$
M_{n}(\mathfrak{Q})=N\left(\mathfrak{A} \vee f^{-1}(\mathfrak{A}) \vee \cdots \vee f^{-n}(\mathfrak{P})\right) .
$$

Here $f^{-1}(\mathbb{G})$ denotes the open cover $\left\{f^{-1}(A) \mid A \in \mathbb{U}_{\}}\right.$, and $f^{n}$ is defined inductively by $f^{1}=f$ and $f^{n}=f^{n-1} \circ f$ for $n>1$.

Set

$$
\operatorname{ent}(f, \mathfrak{A})=\lim _{n \rightarrow \infty} n^{-1} \log M_{n}(\mathfrak{A})
$$

It is easy to show that this limit exists and is finite (see [1]). Finally we define the topological entropy of $f$ by ent $(f)=\sup$ ent $(f, \mathbb{A})$ where the supremum is taken over all open covers $\mathbb{A}$ of $X$.

Next we define the notion of nonwandering set. Let $f \in C^{0}(X, X)$. A point $x \in X$ is said to be wandering if there is a neighborhood 0 of $x$ such that $f^{n}(0) \cap 0=\varnothing$ for each integer $n>0$. The set of points which are not wandering is called the nonwandering set and denoted $\Omega(f)$. We remark that $\Omega(f)$ is a closed subset of $X$ and $f(\Omega(f)) \subset \Omega(f)$.

The following proposition is proved by Bowen in [4]. Here $X$ is a compact metric space.

Proposition 1. Let $f \in C^{0}(X, X)$. Then ent $(f)=\operatorname{ent}(f \mid \Omega(f))$.

One of the inequalities necessary for Proposition 1 follows immediately from the following basic fact which is proved in [1].

Proposition 2. Let $f \in C^{0}(X, X)$ and let $K$ be a closed subset of $X$ 
such that $f(K) \subset K$. Then ent $(f) \geq$ ent $(f \mid K)$.

It follows immediately from the definition that if $K$ is finite and $f \epsilon$ $C^{0}(K, K)$ then ent $(f)=0$. Hence by Proposition 1 we have

Proposition 3. Let $f \in C^{0}(X, X)$. If $\Omega(f)$ is finite then ent $(f)=0$.

From the definition of $\Omega(f)$ it follows that $\Omega(f) \subset \operatorname{Im}(f)$ (the image of $f$. Hence we have

Proposition 4. Let $f \in C^{0}(X, X)$. If $\operatorname{Im}(f)$ is finite then ent $(f)=0$.

3. Proof of Theorem A. We may think of the Cantor set $C$ as the set of infinite sequences $\left(x_{1}, x_{2}, \ldots\right)$ such that each $x_{k}$ is 1 or 2 . The topology on $C$ is then given by the metric

$$
d\left(\left(x_{1}, x_{2}, \cdots\right),\left(y_{1}, y_{2}, \cdots\right)\right)=\sum_{i=1}^{\infty}\left(2^{-i}\right)\left|x_{i}-y_{i}\right| .
$$

(Equivalently we are thinking of $C$ as the infinite product of the set $\{1,2\}$ with the product topology.)

Let $f \in C^{0}(C, C)$. We have two cases.

Case 1. ent $(f)>0$.

Define a sequence $\left(f_{k}\right)$ of functions in $C^{0}(C, C)$ as follows. Let

$$
f_{k}\left(x_{1}, x_{2}, \cdots\right)=\left(y_{1}, y_{2}, \cdots, y_{k-1}, y_{k}, 1,1,1, \cdots\right)
$$

where $f\left(\dot{x}_{1}, x_{2}, \cdots\right)=\left(y_{1}, y_{2}, \cdots\right)$. In other words $f_{k}$ is the function which assigns to a sequence $\left(x_{1}, x_{2}, \ldots\right)$ the sequence whose first $k$ terms are the first $k$ terms of $f\left(x_{1}, x_{2}, \ldots\right)$ and whose terms past the $k$ th term are all 1 .

Note that the image of the map $f_{k}$ is a finite set consisting of at most $2^{k}$ points. (For example the image of $f_{2}$ consists at most of the points $(1,1,1,1,1, \ldots),(1,2,1,1,1, \ldots),(2,1,1,1,1, \ldots)$, and $(2,2,1,1,1, \ldots)$. Hence by Proposition 4 , ent $\left(f_{k}\right)=0$.

It is easy to see that the sequence $\left(f_{k}\right)$ converges uniformly to $f$. In fact if $\epsilon>0$, we can choose an integer $N$ large enough to insure that $\left(\sum_{k=N+1}^{\infty} 2^{-k}\right)<\epsilon$. Then for $k \geq N$,

$$
d\left(f_{k}\left(x_{1}, x_{2}, \cdots\right), f\left(x_{1}, x_{2}, \cdots\right)\right)<\epsilon
$$

for any $\left(x_{1}, x_{2}, \ldots\right) \in C$.

Case 2. ent $(f)=0$.

Define a sequence $\left(g_{k}\right)$ of functions in $C^{0}(C, C)$ as follows. Let $f\left(x_{1}, x_{2}, \ldots\right)=\left(y_{1}, y_{2}, \ldots\right)$ and set 


$$
g_{k}\left(x_{1}, x_{2}, \cdots\right)=\left(y_{1}, y_{2}, \cdots, y_{k-1}, y_{k}, x_{k+2}, x_{k+3}, \cdots\right) .
$$

In other words $g_{k}\left(x_{1}, x_{2}, \ldots\right)$ is the sequence whose first $k$ terms are the same as the first $k$ terms of $f\left(x_{1}, x_{2}, \ldots\right)$, and whose $n$th term for $n>k$ is $x_{n+1}$.

As in Case 1 , it is clear that $\left(g_{n}\right)$ converges uniformly to $f$. We conclude the proof by showing that for each integer $k>0$, ent $\left(g_{k}\right) \geq \log (2)$. Fix $k>0$.

Let $O_{1}$ be the set of sequences $\left(x_{1}, x_{2}, \ldots\right)$ such that $x_{k+1}=1$. Let $\mathrm{O}_{2}$ be the set of sequences $\left(x_{1}, x_{2}, \ldots\right)$ such that $x_{k+1}=2$. Then $\mathbb{A}=$ $\left\{O_{1}, O_{2}\right\}$ is an open cover of $C$. We will show that ent $\left(g_{k}, \mathbb{Q}\right)=\log (2)$.

Let $x=\left(x_{1}, x_{2}, \cdots\right) \in C$. Then

$$
\begin{aligned}
& x \in O_{1} \cap g_{k}^{-1}\left(O_{1}\right) \Leftrightarrow x_{k+1}=1 \text { and } x_{k+2}=1, \\
& x \in O_{1} \cap g_{k}^{-1}\left(O_{2}\right) \Leftrightarrow x_{k+1}=1 \text { and } x_{k+2}=2, \\
& x \in O_{2} \cap g_{k}^{-1}\left(O_{1}\right) \Leftrightarrow x_{k+1}=2 \text { and } x_{k+2}=1, \\
& x \in O_{2} \cap g_{k}^{-1}\left(O_{2}\right) \Leftrightarrow x_{k+1}=2 \text { and } x_{k+2}=2 .
\end{aligned}
$$

Thus the sets $O_{1} \cap g_{k}^{-1}\left(O_{1}\right), O_{1} \cap g_{k}^{-1}\left(O_{2}\right), O_{2} \cap g_{k}^{-1}\left(O_{1}\right)$, and $\mathrm{O}_{2} \cap g_{k}^{-1}\left(\mathrm{O}_{2}\right)$ are pairwise disjoint nonempty subsets of $C$. Hence $M_{1}\left(g_{k}, \mathbb{A}\right)=4$. It follows in the same way by induction that $M_{n}\left(g_{k}, \mathbb{A}\right)=$ $2^{n+1}$ for each integer $n>0$. Hence ent $\left(g_{k}, \mathbb{Q}\right)=\log (2)$. This implies that ent $\left(g_{k}\right) \geq \log (2)$, and completes the proof of Theorem $A$.

We remark that since the diameter of $\mathbb{Q} \vee g_{k}^{-1}(\mathbb{A}) \vee \cdots \vee g_{k}^{-n}(\mathbb{A})$ approaches zero (as $n \rightarrow \infty)$, it actually follows that ent $\left(g_{k}\right)=\log (2)$.

4. Proof of Theorem B. Let $K$ denote any closed interval on the real line. We may form the middle third Cantor subset of $K$, which we denote by $C$, and we may identify points in $C$ with sequences whose terms are all 1 or 2 , as in $\$ 3$.

Let $s$ denote the map in $C^{0}(C, C)$ defined by $s\left(x_{1}, x_{2}, x_{3}, \ldots\right)=$ $\left(x_{2}, x_{3}, x_{4}, \cdots\right) . s$ is sometimes called the full 2-shift (see [8] for discussion and further references). We will use the following elementary facts (see [1]).

Proposition 5. ent $(s)=\log (2)$.

Proposition 6. If $f \in C^{0}(X, X)$ for any compact space $X$, then ent $\left(f^{n}\right)=$ $n \cdot \operatorname{ent}(f)$. 
We will use the usual metric $d$ on $C^{0}(I, I)$ which may be defined by

$$
d(f, g)=\sup \{|f(x)-g(x)|: x \in I\} \text {. }
$$

Theorem B. The function ent: $C^{0}(I, I) \rightarrow R \cup\{\infty\}$ is not continuous at any map $f$ with ent $(f)$ finite.

Proof. Let $f \in C^{0}(I, I)$ with ent $(f)$ finite. Let ent $(f)=\log (K)$. Pick an integer $m>0$, such that $2^{m}>2 K$.

Let $x_{0}$ be a fixed point of $f$. We assume for simplicity that $x_{0} \neq 1$. (The proof can be easily modified for the case $x_{0}=1$.)

Let $\epsilon>0 . \exists \delta>0$ such that if $\left|x-x_{0}\right| \leq \delta$ then $\left|f(x)-x_{0}\right|<\epsilon / 2$. We may choose $\delta$ so that $\delta<\epsilon / 2$ and $x_{0}+\delta \leq 1$.

We construct a map $g \in C^{0}(I, I)$ such that $d(f, g)<\epsilon$. We first construct $g$ on the interval $\left[x_{0}, x_{0}+\delta / 2\right]$ as follows. Let $C$ denote the middle third Cantor subset of the interval $\left[x_{0}, x_{0}+\delta / 2\right]$. Define $g$ on $C$ by $g=s^{m}$, where $s$ denotes the full 2-shift as defined above. Note that $g\left(x_{0}\right)=x_{0}$ and $g\left(x_{0}+\delta / 2\right)=x_{0}+\delta / 2$ since $x_{0}$ is identified with the sequence $(1,1,1, \ldots)$ and $x_{0}+\delta / 2$ is identified with the sequence $(2,2,2, \cdots)$. We extend $g$ to the interval $\left[x_{0}, x_{0}+\delta / 2\right]$ by defining $g$ linearly on each open interval in $\left[x_{0}, x_{0}+\delta / 2\right]-C$.

Next we extend $g$ to the interval $\left[x_{0}, x_{0}+\delta\right]$ by defining $g$ on the interval $\left[x_{0}+\delta / 2, x_{0}+\delta\right]$ as follows. Let $g\left(x_{0}+\delta / 2\right)=x_{0}+\delta / 2$, $g\left(x_{0}+\delta\right)=f\left(x_{0}+\delta\right)$, and define $g$ linearly on $\left[x_{0}+\delta / 2, x_{0}+\delta\right]$. Finally we extend $g$ to a map in $C^{0}(I, I)$ by defining $g(x)=f(x)$ for $x \in I-$ $\left[x_{0}, x_{0}+\delta\right]$.

Note that

$$
\text { ent }(g) \geq \text { ent }\left(s^{m}\right)=\log \left(2^{m}\right)>\log (2 K)=\log (2)+\log (K) \text {. }
$$

We must show that for all $x \in I,|f(x)-g(x)|<\epsilon$.

If $x \in I-\left[x_{0}, x_{0}+\delta\right]$ then $|f(x)-g(x)|=0$. If $x \in\left[x_{0}, x_{0}+\delta\right]$ then

$$
|g(x)-f(x)|<\left|g(x)-x_{0}\right|+\left|f(x)-x_{0}\right|<\epsilon / 2+\epsilon / 2=\epsilon .
$$

Here we have used the fact that $g$ is defined linearly on $\left[x_{0}+\delta / 2, x_{0}+\delta\right]$, and $\left|g\left(x_{0}+\delta / 2\right)-x_{0}\right|<\epsilon / 2$, and $\left|g\left(x_{0}+\delta\right)-x_{0}\right|<\epsilon / 2$.

We have constructed a map $g \in C^{0}(I, I)$ such that $d(f, g)<\epsilon$, and ent $(g)>$ ent $(f)+\log (2)$. Since $\epsilon$ was arbitrary this completes the proof that ent is not continuous at $f$. Q.E.D.

We conclude this section by remarking that Theorem $B$ is valid with $I$ replaced by the circle $S^{1}$. We use the fact that a dense set of maps in 
$C^{0}\left(S^{1}, S^{1}\right)$ have periodic points (see [3]).

Let $f \in C^{0}\left(S^{1}, S^{1}\right)$ and $\epsilon>0$. Let $f_{1} \in C^{0}\left(S^{1}, S^{1}\right)$ such that $f_{1}$ has a periodic point and $d\left(f, f_{1}\right)<\epsilon / 2$. By modifying the argument of Theorem $\mathrm{B}$, with a periodic orbit replacing the role of the fixed point, we construct a map $g$ with ent $(g)>$ ent $(f)+\log (2)$, and $d\left(f_{1}, g\right)<\epsilon / 2$. Hence ent is not continuous at $f$.

5. An example. We close by giving an example of $f \in C^{0}(I, I)$ such that ent $(f)$ is infinite.

Let $K_{n}$ denote the interval $[1 /(n+1), 1 / n]$ for each integer $n>0$. Define $f$ on each interval $K_{n}$ as follows. Let $C_{n}$ denote the middle third Cantor subset of $K_{n}$. Let $f=s^{n}$ on $C_{n}$ (again $s$ denotes the full 2-shift defined in $\$ 4$ ) and extend $f$ to $K_{n}$ by defining $f$ linearly on each open interval in $K_{n}-C_{n}$. We extend $f$ to a map in $C^{0}(I, I)$ by setting $f(0)=0$.

It follows from Propositions 2,5 , and 6 that ent $(f)=\infty$.

\section{REFERENCES}

1. R. L. Adler, A. G. Konheim and M. H.'McAndrew, Topological entropy, Trans. Amer. Math. Soc. 114 (1965), 309-319. MR 30 \#5291.

2. L. Block, Bifurcations of endomorphisms of $S^{1}$, Thesis, Northwestern University, 1973.

3. L. Block and J. Franke, Existence of periodic points for maps of $S^{1}$, Invent. Math. 22 (1973), 69-73.

4. K. Bowen, Topological entropy and axiom A, Proc. Sympos. Pure Math., vol. 14, Amer. Math. Soc., Providence, R. I., 1970, pp. 23-41. MR $41 \# 7066$.

5. - Entropy for group endomorphisms and homogeneous spaces, Trans. Amer. Math. Soc. 153 (1971), 401-414. MR 43 \#469.

6. M. Misiurewicz, On non-continuity of topological entropy, Bull. Acad. Polon. Sci. Sér. Sci. Math. Astronom. Phys. 19 (1971), 319-320. MR 44 \#4781.

7. M. Shub, Dynamical systems, filtrations, and entropy, Bull. Amer. Math. Soc. 80 (1974), 27-41.

8. S. Smale, Differentiable dynamical systems, Bull. Amer. Math. Soc. 73 (1967), 747-817. MR 37 \#3598; erratum, 39, p. 1593.

DEPARTMENT OF MATHEMATICS, UNIVERSITY OF FLORIDA, GAINESVILLE, FLORIDA 32611 